\title{
BIOÉTICA EN LA EXPERIMENTACIÓN CON SERES HUMANOS
}

José Miguel Esquivel

En la práctica de la medicina se utilizan los conocimientos con un determinado y concreto fin: prevenir, aliviar o curar los padecimientos de un ser humano enfermo. Para este fin, se han de tomar y usar conocimientos de la fisiología, de la anatomía, de la bioquímica, de la microbiología, de la genética, de la medicina nuclear, de la inmunología, de la patología etc., pero también de la física, de la química, etc. y aún más, de la psicología, de la sociedad y también de la ética. Lo anterior implica que se debería hablar de ciencias médicas y biomédicas, más que de medicina simplemente. Lo que debe subrayarse, es la visión global, la concepción holística del paciente. Esto plantea una progresiva subdivisión y superespecialización del saber médico, generando problemas de orden epistemológico-didáctico e incluso de orden ético. 
Hace aproximadamente 50 años, la ética médica era, como lo había sido durante siglos, un dominio circunscrito a la profesión médica, protegida de los cambios culturales y enmarcada dentro de preceptos morales que parecían inmutables. En efecto, a pesar de la revolución tecnológica biomédica posterior a la Segunda Guerra Mundial, lo único que parecía inalterable en medio de la metamorfosis que afectaba a la medicina en todos sus aspectos, era la ética médica.

En la actualidad, las normas del juramento de Hipócrates y la ética médica son, obviamente, insuficientes para poder resolver los dilemas relacionados con el desarrollo de la ciencia, la tecnología biomédica y los seres humanos. Este marco de referencia está padeciendo la más severa tensión de su historia. La ética médica se ha transformado en objeto de la más amplia preocupación pública. Cada una de las doctrinas derivadas de la interpretación del juramento hipocrático y la ética médica, está siendo cuestionada y al mismo tiempo, reformulada. El desarrollo de la cultura universal ha provocado conflictos morales que han desintegrado los valores tradicionales. Esta transformación tiene causas sociales, políticas, económicas y jurídicas. Sin embargo, ninguna de éstas es más importante que el hecho de que la totalidad de la ética médica esté siendo objeto de indagación filosófica en el nivel mundial.

En la cultura occidental, algo que no podemos dejar de lado es el predominio de la religión en los aspectos éticos que influyeron en las ciencias médicas. Su importancia podría haber variado, pero nunca ha estado ausente. La evidencia de esto se halla al analizar el enfoque de los problemas médicos que surgen de la relación médico y paciente, desde el punto de vista de tres de las grandes religiones: catolicismo, judaísmo y protestantismo. Los temas de interés son 
los mismos en cada una de ellas: preservación de la vida, preparación para la muerte, aborto, esterilización, eugenesia, eutanasia, inseminación artificial, medidas anticonceptivas y manipulación genética.

\section{Bioética}

La bioética surge como respuesta al impacto del desarrollo de la biología molecular, en la evolución del hombre. El término nace, con la implementación de las técnicas de manipulación del genoma humano, conocida como ingeniería genética.

En 1973, Van Rensselaer Potter, creador del neologismo bioético, lo proyecta como una interdisciplina que combinará el conocimiento biológico con el conocimiento de los sistemas de valores humanos: "Elegí bio para representar el conocimiento biológico, la ciencia de los sistemas vivientes y elegí ética para representar el conocimiento de los sistemas de valores humanos. (17). En la génesis, la bioética plantea una nueva disciplina que reflexionaría de manera racional y científica sobre problemas morales de las ciencias biológicas y de la medicina y respondería de manera científica a los desafíos de los descubrimientos científicos y tecnológicos en las ciencias biológicas y propondrían soluciones racionales y prácticas. El significado de "bioética" según la Real Academia Española XXI edición: "Disciplina científica que estudia los aspectos éticos de la medicina y la biología en general, así como de las relaciones del hombre con los restantes seres vivos".

Desde hace 50 años, como fenómeno universal, se da un desarrollo científico y tecnológico desmesurado, junto a una erosión moral en la sociedad y por lo tanto, los fundamentos 
morales tradicionales y las ciencias médicas son puestos en duda. Muchos médicos trataron de escudarse en el ordenamiento jurídico perdiendo la confianza en la ética tradicional como guía para la toma de decisiones morales. De esta manera, se crearon modelos alternos para la enseñanza y práctica de la ética médica.

Bioética es una rama del saber ético, un estudio de la realidad del cuidado de la salud, basado en datos de las ciencias de la vida como la biología, la medicina, la antropología, la sociología y más recientemente las humanidades. Tiene una metodología interdisciplinaria que comprende el derecho y la política. La perspectiva que domina proviene de una evolución histórica desde la Segunda Guerra Mundial, no solamente de la ciencia y tecnología, sino de la democratización de las relaciones sociales y de la desconfesionalización de la ética tradicional. Se opone a la ética médica (tradición hipocrática) dominada durante siglos por la reflexión teológica en cuestiones "estrictamente" médicas. Por oposición a la ética médica tradicional, valoriza, no solamente aproximaciones filosóficas sino aproximaciones científicas, basadas en metodologías empíricas y cuantitativas, como la epidemiología clínica y los cuestionamientos de las ciencias sociales.

En la genética de la bioética ha influido un conjunto de acontecimientos, de los que destacamos tres: $(15,21)$

- La ingeniería genética, las técnicas de reproducción humana, los transplantes de órganos, los progresos técnicos en la práctica de la reanimación, la eutanasia, la distanasia, el aborto, la esterilización, la contracepción, el genoma humano.

- Los cambios operados en el concepto de la salud y en la práctica médica, la extrema tecnificación instrumental y 
una peculiar actitud del médico ante ella. La colectivización de la asistencia médica en todos los países del globo, la despersonalización del enfermo, la prevención de la enfermedad, la promoción de la salud y el problema de si es técnicamente posible una mejora de la naturaleza humana.

- Desconfesionalización y no ontologización de la ética, es decir que la bioética ha de apoyarse en la racionalidad humana, secular y compartida por todas las personas y que ha de situarse en el terreno filosófico, buscando como paradigma la racionalidad ética. La bioética comprende los conflictos relacionados con principios morales que surgen en las profesionales de la salud, incluso en las profesiones afines y vinculadas con la salud mental, así como las aplicaciones de la ciencia que afectan el curso de la vida. Se aplica a las investigaciones biomédicas y clínicas y sobre el comportamiento, independientemente de que influya o no de forma directa en la terapéutica. Aborda cuestiones sociales, como las que se relacionan con la salud pública, la salud ocupacional y la ética del control político de la natalidad, entre otras. Va más allá de la vida y la salud humana, en cuanto comprende cuestiones relativas a la vida de los animales y las plantas. (15)

\section{Experimentación en seres humanos}

La historia de la humanidad consigna la experimentación en seres humanos en las civilizaciones conocidas. Para descubrir la cura de diferentes dolencias, tuvo que ensayarse en seres humanos hasta encontrar un tratamiento adecuado para cada enfermedad. Aristóteles destacó la importancia de la experimentación en seres humanos vivos, para los conocimientos sobre el funcionamiento del organismo. En la 
Alejandría de Ptolomeo y otros lugares de Oriente, se usaba a los prisioneros y los condenados a muerte para llevar a cabo experimentaciones. En la Europa Medieval, los monarcas autorizaban la utilización de condenados a muerte para la investigación.

En el siglo XX, los medios de comunicación publicaron, rara vez, información sobre experimentos en humanos, porque era un tema reservado a la elite de los científicos. No fue hasta que se hicieron públicos los informes sobre los actos de experimentación en prisioneros en Alemania y Japón, en la Segunda Guerra Mundial, que la atención mundial conoció en detalle sobre la investigación clínica que se envía dando desde hacía muchos años en varios países.

Luego salieron a relucir informes sobre investigación clínica en Estados Unidos, algunos financiados con fondos públicos, entre ellos el estudio de la sífilis en Tuskegee, Alabama, el cual salió a la luz pública en 1972, no obstante haberse iniciado en 1932. La experimentación la organizó el Servicio de Salud Pública de los Estados Unidos, para establecer prospectivamente la historia natural de la sífilis no tratada en negros. Aunque en 1942 comenzó la comercialización de la penicilina, los participantes en el experimento no fueron tratados con ella. Tuvieron que sobrellevar la enfermedad hasta su muerte, aunque ya se conocía un tratamiento eficaz. La historia fue denunciada en 1972 por un periodista, lo que obligó al gobierno a ordenar una investigación, que culminó en un proceso judicial en el que las víctimas del experimento y sus descendientes fueron indemnizados. A principios del año 1999 , el presidente Clinton pidió perdón públicamente a las familias afectadas.

El primer documento internacional sobre la ética de la investigación se promulgó en 1947, como consecuencia 
del juicio a los médicos que habían realizado experimentos con prisioneros sin su consentimiento. Este documento, conocido como Código de Nuremberg, tiene un único objetivo, proteger la dignidad de la persona sometida a experimentos médicos o científicos.

La Declaración de Helsinki, promulga en 1964 por la Asociación Médica Mundial y subsiguientes revisiones, la última en octubre del 2000 en Edimburgo, Escocia, es fundamental en el campo de la ética de la investigación biomédica y constituye hoy día el documento de referencia obligado para la formulación de la legislación internacional y nacional.

Para otorgar fuerza jurídica y moral a la Declaración Universal de los Derechos Humanos, adoptada por la Asamblea General de las Naciones Unidas en 1948, la Asamblea General aprobó en 1966, el Acuerdo Internacional sobre los Derechos Civiles y Políticos cuyo artículo 7 expresa lo siguiente: "Nadie será sometido a tortura o a un tratamiento o castigo cruel, inhumano o degradante. En especial, nadie será sometido sin su libre consentimiento a experimentación médica o cientifica".

En 1982 la Organización Mundial de la Salud y el Consejo de Organizaciones Internacionales de Ciencias Médicas (CIOMS), propusieron las Pautas internacionales para la Evaluación Ética de los Estudios Epistemológicos y Experimentación Biomédica, texto que fue revisado en 1993 (6). Estas pautas tienen como objetivo señalar la aplicạción de los principios éticos que deben regir la ejecución de la investigación en seres humanos, especialmente para países en desarrollo, según lo establecido en la Declaración de Helsinki y la aplicabilidad del Código de Nuremberg. 
La investigación clínica (clinical trials) incluye diversos estudios concernientes a la salud humana. Los avances en atención médica y prevención de enfermedades van a depender de la forma en que se entienden los procesos fisiológicos y patológicos, o los resultados de los estudios epidemiológicos, los cuales necesitan experimentación en seres humanos. Los resultados obtenidos de muchas investigaciones contribuyen, en forma apreciable, al mejoramiento de la salud humana. $(1,2,3,4,5,6,7)$.

Existen principios bioéticos universalmente aceptados para guiar el trabajo de investigador, entre muchos otros los que a continuación se citan a manera de ejemplo, $(6,7)$. El desconocerlos viola los Derechos Humanos y constituye pseudociencia.

- El respeto a las personas (autonomía, consentimiento informado): quienes van a ser participantes de un estudio deben estar informados adecuadamente. El respeto se refleja en no hacerles promesas falsas, en mantener su identidad reservada cuando las circunstancias lo requieran y saber que cada uno tiene una historia de vida a la que le debemos el mayor respeto.

- La búsqueda del bien: todo acto médico debe ser benéfico para el paciente, el cual es el que necesita los servicios de salud. Esto hace que los investigadores y las profesionales en general tengan una gran responsabilidad.

- $\quad$ No hacer daño. "Primum non nocere" (Hipócrates).

- La justicia distributiva: los beneficios deben ser todos, independientemente de su participación. Así, es obligatorio que los resultados obtenidos sean un bien público. La honestidad, "evitar el engaño", es un requisito para todo investigador para no engañar o autoengañarse. 
Hay que tener presente que la metodología de la investigación clínica se inicia elaborando hipótesis que son sometidas a pruebas de laboratorio, con animales de experimentación y para poder obtener conclusiones útiles clínicamente, se debe realizar el experimento en humanos. Este tipo de investigación, aunque se diseñe con el máximo cuidado, siempre entraña riesgo para un ser humano. El riesgo se justifica, no porque signifique un beneficio pecuniario para el investigador o la institución investigadora, sino más bien porque beneficiaría a las personas participantes y porque habría una posible contribución al conocimiento humano.

Los proyectos de investigación deben contar con certificación que garantice respetar las reglas éticas y que demuestre que el protocolo ha sido aprobado por un Comité de Ética. La función principal de este comité es velar por los derechos de las personas participantes en una investigación. $(10,12,13,17)$.

El problema ético en la investigación en humanos, se inicia cuando la persona se torna en un laboratorio viviente que recibe una información incompleta, no comprensible para ella o que por su situación social, económica o mental favorece que se convierta en un candidato especial para esos experimentos.

Las personas que van a participar en un estudio deben ser libres para decidir si lo hacen o no, luego de que hayan recibido información clara, adecuada y suficiente para saber de qué se trata el estudio. Esto es la base del consentimiento informado y deben considerarse tres posibilidades. 
1. Toma de decisión consciente: se debe dar toda la información posible sobre los riesgos, beneficios, métodos, duración, derechos de la persona, sistema de elección, etc.

2. Consentimiento libre: dar un tiempo prudencial a la persona para que reflexione y esté segura de si desea participar o no.

3. Consentimiento claramente expresado: se debe dar por escrito y si existe alguna dependencia entre el sujeto y el investigador, es mejor que haya una tercera persona neutral que escuche la explicación $(6,7)$.

\section{Los comités hospitalarios de ética}

Los comités hospitalarios de ética son grupos interdisciplinarios, con las funciones de docencia, investigación y consulta, asociados con los dilemas éticos que surgen durante la práctica de la medicina. Esta figura institucional tiene su origen en la problemática de las nuevas tecnologías médicas y en una nueva conciencia social orientada hacia la evaluación de tratamientos prolongados, decisiones de no tratamiento en recién nacidos con malformaciones graves, investigaciones en seres humanos, (prematuros, lactantes, mujeres embarazadas, pacientes psiquiátricos) y otras deliberaciones de índole ético ya citados.

Se debe tomar en cuenta el indiscutible papel interdisciplinario que presenta la bioética que incluye, para poder satisfacer sus requerimientos, a distintos profesionales de la salud, a filósofos, teólogos, representantes de la comunidad y juristas, quedando todo reflejado en los comités de bioética, en donde se representan los valores e intereses necesarios para la toma de decisiones que así lo requieran. 
En 1976, el juez Richard Hughes de la Corte Suprema de Nueva Jersey, EE.UU., respondió a la demanda de suspender la respiración artificial de una joven (Karen Quinlan), en estado de coma profundo, señalando que debía consultarse al comité de ética del hospital en el que se hallaba internada. Hughes apelaba, entre los fundamentos de su juicio, a un artículo reciente de una pediatra, Karen Teel, en el que ésta proponía un comité de ética integrado por médicos, trabajadores sociales, abogados y teólogos, como instrumento de diálogo para la evaluación de diferentes opciones terapéuticas aplicables a un determinado paciente. Se inicia de esta forma la historia de los comités de bioética. $(4,22)$.

Treinta años del caso Quinlan, la otra historia comenzaba. En 1946, un tribunal internacional constituido en Nuremberg, condenaba a miembros del partido nazi por crímenes contra la humanidad. El año siguiente, tras haber descubierto los experimentos llevados a cabo con prisioneros de guerra, el tribunal proclamaba el Código de Nuremberg que estableció un decálogo de principios que deberían ser respetados en casos de experimentación médica sobre seres humanos. Este código se utilizó como base para el Código de Ética en Experimentación Humana redactado por la Asociación Médica Mundial y desarrollado por la Declaración en Helsinki de la Asamblea Médica Mundial, se recomienda expresamente que cada fase de la experimentación con sujetos humanos, debe someterse a la consideración de un comité independiente especialmente constituido a tal efecto. En 1975, la Dra., Karen Teel, publica su famoso artículo ya citado y en 1981, la Organización Mundial de la Salud, difundió la Propuesta de Normas internacionales para la investigación biomédica en sujetos humanos, destinada a indicar la conveniencia de aplicar la Declaración de 
Helsinki revisada en Tokio, específicamente para los países en desarrollo, conocidas como las Pautas del CIOMS. La última enmienda a la Declaración de Helsinki, se realizó en la $52^{3}$ Asamblea General de la Asociación Médica Mundial en Edimburgo, Escocia, en octubre del 2000.

\section{La bioética en Costa Rica}

De acuerdo con los archivos de la Asamblea Legislativa de Costa Rica, se puede inferir que el desarrollo y la aplicación en nuestro país del conocimiento y aplicación de la bioética, es casi nulo.

Miles de personas han participado, sin saberlo, en experimentos de ensayos de medicamentos experimentales. Posibles abusos en la investigación clínica están enfrentados a la población nacional a nuevos problemas éticos, pues se están empleando seres humanos enfermos de hospitales, para probar medicamentos y vacunas de los cuales aún no se tiene evidencia científica suficiente de su eficacia terapéutica o se pretende experimentar modificaciones de las indicaciones terapéuticas conocidas de algunos medicamentos; y Costa Rica como es conocido, carece de controles sanitarios estrictos y de una legislación actualizada, en esta materia.

Así por ejemplo, en 1976, un instituto de investigación patrocinado por la Universidad de Louisiana, EE.UU., el ICMRT, implementó 40 protocolos de investigación en niños y adultos sin el permiso del Ministerio de Salud. Los protocolos habían sido aprobados por un comité de profesionales en ciencias médicas, abogados, un clérigo, sin conocimiento de las normas internacionales sobre investigación clínica y aparentemente sin obtener los respectivos consentimientos informados, tal y como lo establece la Declaración de Helsinki. 
El interés del centro fue experimentar vacunas, algunas de ellas vencidas y drogas que no podrían ensayarse fácilmente en otros países, por dificultarlo su propia legislación y realizar cientos o miles de pruebas en seres humanos supuestamente "voluntarios", que no se percataron y no fueron informados que serían utilizados y de este modo, obtener la autorización del F.D.A., EE.UU., para la comercialización y venta del producto.

En 1986, el Dr. Alfonso Trejos Willis (investigador y profesor de la Universidad de Costa Rica), denunció: "Esta actividad, (la investigación clínica) si bien es científica y éticamente espuria resultó sumamente lucrativa, tanto así que en un plazo de 10 años desde su fundación, el LSU. ICMRT dedicó todo su personal a este tipo de "investigación" y finalmente llegó a tener un presupuesto financiado en un $79 \%$ por la casa Merck Sharp and Dohme Inc." (18).

De acuerdo con la tesis de grado para optar al título de Licenciada en Derecho de la Universidad de Costa Rica, "La tutela constitucional del derecho a la vida y a los actos de experimentación sobre seres humanos", de Marisol Rodríguez Cordero, la historia de la experimentación biomédica en Costa Rica tiene un protagonista principal: el ICMRT (International Center for Medical Research and Training). $(18,21)$. En 1976, la Asamblea Legislativa integró una comisión especial que investigara una vacunación experimental en tres guarderías, con el virus sincicial respiratorio, sin el consentimiento de los padres y de haber realizado una vacunación masiva en Mozotal de Guadalupe, con una vacuna experimental contra el sarampión, no autorizada por el Ministerio de Salud. Fueron llevados a los Tribunales pero la causa fue desestimada, por no estar tipificada como delito en nuestro actual Código Penal. 
Señala Rodríguez Cordero en su tesis: "El Ministerio de Salud se había opuesto a que se incluyeran disposiciones sancionatorias en esta materia pues, según manifestó, la legislación existente cubría en lo que era necesario las posibilidades infracciones de los experimentadores. Discrepamos de la opinión que entonces exteriorizó el Dr. Weinstok. No está de más indicar lo que señala el Ministro (aunque esté sobreentendido que un permiso administrativo y una autorización de la persona sometida a experimentación no puede suponer la renuncia de derechos consagrados por el ordenamiento ni mucho menos la creación de una especie de fuero especial en el que no sea de aplicación el derecho penal), pero consideramos que la creación de sanciones en la materia de experimentación sobre seres humanos se justifica por lo delicado de la materia. Ya en 1976 se tuvo la experiencia del ICMRT, que desobedecio reiteradamente las normas sobre investigación biomédica y no hubo forma de que prosperara la acción penal contra sus directores, simplemente porque el derecho penal es de aplicación restrictiva y se necesita que un tipo contenga la descripción exacta de la conducta juzgada para proceder a la imposición de las penas. Pensamos que eventualmente disposiciones penales podrian persuadir al investigador de cometer abusos en las experimentaciones." (21).

La Asamblea Legislativa aprobó en 1976 una reglamentación sobre experimentación en seres humanos, que nunca llegó a convertirse en ley porque el Poder Ejecutivo la vetó y no fue resellada, aduciendo entre otras cosas, que la Ley General de Salud era suficiente para regular la experimentación en humanos, hecho totalmente rebatido hoy día, por la fuerza de los acontecimientos y del desarrollo de la ciencia y tecnología biomédica. El proyecto fue archivado definitivamente en 1986.

Es paradójico que en nuestro país exista una ley específica que dedica un capítulo a los experimentos con animales 
(Ley de Bienestar de los Animales, \#7451) y no exista una ley que, al menos, dedique un capítulo entero a sistematizar las disposiciones relativas a la experimentación en seres humanos. Así los hechos, las condiciones de pobreza de los países en vías de desarrollo y la falta de una ley específica, favorecen los ensayos de medicamentos por parte de compañías multinacionales productoras de medicamentos.

En 1998, se publicó el Decreto №. 27349.S, Reglamentos para la investigación de seres humanos, el cual elimina el decreto 5463-SPPS de 1975 y el decreto 24396-S de 1995, siendo el cuerpo normativo con más cantidad de disposiciones sobre la experimentación en seres humanos. Antes de la publicación de este decreto, el Comité Bioética y la Dirección Jurídica de la CCSS, emitieron criterio negativo basado en consideraciones de forma y fondo, pues fue aparentemente redactado con la participación de los reclutadores de pacientes de una empresa privada que se dedica, a exportar "investigación científica" a empresas farmacéuticas de los Estados Unidos y Europa. En realidad lo que hace, según los archivos de la CCSS, es reclutar pacientes de los hospitales de esta institución, para probar medicamentos en fase experimental de compañías transnacionales, utilizando protocolos de científicos extranjeros. $(4,9)$. Dicho decreto tiene todos los vicios y defectos de un cuerpo normativo, cuyo objetivo es realizar investigación para probar medicamentos, desde la perspectiva de los reclutadores de pacientes, dejando de lado a la normativa internacional, que salvaguarda y protege los derechos humanos de los pacientes.

Hay evidencia escrita en la Asamblea Legislativa y en la Caja Costarricense de Seguro Social (CCSS), de que en los últimos 30 años se han desarrollado muchos protocolos de ensayos clínicos, (más de quinientos), redactados en el 
extranjero, subvencionados por compañías transnacionales fabricantes de medicamentos, por lo que los científicos costarricenses se convirtieron en reclutadores de pacientes. Mientras tanto, la masa de investigadores productivos permaneció exigua, pocos profesionales concretaron el doctorado académico, las escuelas de ciencias de la salud publicaron muy poco. El grueso de los protocolos de investigación científica, no obedeció a hipótesis de trabajo de problemas de salud costarricenses, sino a protocolos elaborados por científicos extranjeros. Las pocas publicaciones científicas fueron firmadas casi sólo por científicos extranjeros.

Muchos obstáculos al desarrollo y conocimiento de la bioética concurren por el desarrollo institucional y la permanente crisis socioeconómica. A finales de los años 70 , comienza un episodio de parálisis de la ciencia nacional. Sin embargo, en las dos últimas décadas, proliferan varios centros de investigación en el campo de la salud y medicina, sin aumento significativo en la producción per-cápita de investigaciones por año.

La ciencia y la tecnología fue rezagándose, e incluso algunos científicos se convirtieron en exportadores de especímenes para análisis en el extranjero. Otros científicos se dedicaron a extraer muestras de sangre de pacientes con deficiencias mentales o enfermedades genéticas, para ser analizadas en el extranjero con miras a obtener reactivos químicos, medicamentos, etc. y reciben a cambio pago en dinero, a espaldas de las autoridades hospitalarias y universitarias.

Una nueva dificultad nació en 1992, cuando la CCSS y un centro privado que paralelamente obtuvo un fuero de zona franca, (propio de fábricas de artículos electrodomésticos, 
computadores y ropa, conocidas como maquila), firmaron un convenio que autoriza al centro privado a ensayar medicamentos y vacunas en personas enfermas, con las facilidades de la infraestructura hospitalaria del estado (CCSS), recurso humano y financiero. Todo lo anterior bajo el falso ropaje de investigación autóctona. El centro privado no posee infraestructura médica para hospitalizar pacientes, ni académica para estudiar, evaluar y publicar los estudios clínicos.

La alianza plasmada en ese convenio avaló de 100 protocolos clínicos diseñados por científicos extranjeros, para probar medicamentos, en niños y adultos hospitalizados en la CCSS, lo cual permitió ingresos de más de mil millones de colones al centro privado, según informó el semanario de la Universidad de Costa Rica.

En agosto de 1996, la CCSS integra el primer comité de bioética de Costa Rica, de acuerdo con la normativa citada anteriormente, bajo la coordinación académica del Programa Regional de Bioética de la OPS, OMS, con sede en Chile. En 1998, la CCSS aprobó el primer Reglamento para la investigación, cuyo objetivo es aplicar con eficacia los principios éticos en la Investigación con seres humanos. A la fecha, no se aplica. Posteriormente, en diciembre de 1998, la Gerencia Médica disuelve el Comité de Bioética sin explicación alguna, utilizando un acuerdo de Junta Directiva, emitido para otro propósito, sobrepasando la autoridad conferida en la letra y espíritu del acuerdo original. $(4,8,10,11,14)$.

En la actualidad, Costa Rica no tiene comités de bioética, únicamente existen comités científicos, integrados por reclutadores de pacientes, cuyo objetivo es realizar 
investigaciones clínicas financiadas por compañías transnacionales $(4,8)$.

Revisados los archivos de la CCSS, de la Unidad de Investigación y Bioética, existe información parcial sobre las actividades de investigación. Supuestamente fueron eliminados o nunca se consignó esta información, como un proceso normal de seguimiento, donde se utilizaron seres humanos. En el ámbito mundial, se tiene como norma, para proteger los derechos humanos de los pacientes, que el expediente clínico de un paciente que participó en un estudio para ensayar un medicamento no probado (en fase experimental), debe estar custodiado, por lo menos durante 10 años.

Se encontró información a partir de 1992 y hasta diciembre de 1998, la que se resume a continuación:

- Existe registro de 126 procesos de investigación en total

- 123 corresponden a estudios clínicos para probar medicamentos de compañías farmacéuticas transnacionales, de los cuales aún no se tiene evidencia científica suficiente de su eficacia terapéutica.

- En 96 estudios, no hay original, únicamente el título y el nombre del responsable, no existe copia del consentimiento informado otorgado por el paciente, no se sabe cuántos pacientes participaron, no se saben los resultados obtenidos, no existe recomendación ética científica de un comité independiente de ética, no se sabe cuánto pagó la compañía farmacéutica.

- En 17 estudios, existe recomendación ética científica de un Comité nacional, no se sabe cuántos pacientes 
participaron, no hay copia del consentimiento informado otorgado por el paciente, no sabe de los resultados.

- Los tres protocolos restantes son de investigación epidemiológica, de los cuales uno de ellos corresponde a una hipótesis de trabajo costarricense. Existe protocolo original. No se sabe cuántos pacientes participaron, no se saben resultados, hay recomendación ética científica, no hay copia del consentimiento informado otorgado por el paciente.

- Algunos medicamentos estaban en fase 1,2 y 3 y lógicamente, deberían haberse probado primero en los países industrializados donde se habían generado.

- Ninguno de los estudios clínicos tiene copia de la recomendación ética-científica del país sede de la compañía farmacéutica multinacional que lo patrocina.

- Los documentos de la CCSS. consultados crean dudas de que en todos estos estudios se haya obtenido el consentimiento informado del paciente, tal y como lo demanda la normativa internacional.

- Los fondos para hacer los estudios mencionados fueron percibidos por el centro privado mencionado y luego donó el $15 \%$ a una función, de la cual la CCSS no tiene control, según manifestó la Auditoría de esta Institución.

- Las compañías fabricantes de medicamentos transnacionales han aportado más de mil millones de colones al centro privado y un porcentaje, imposible de verificar, ha beneficiado a profesores universitarios, profesionales 
de la salud, administrativos, a cargo del proceso de reclutamiento de pacientes y obtención de muestras en las salas de los hospitales nacionales.

- Para verificar el impacto de estos 126 estudios clínicos de la CCSS, se cotejó con la información obtenida por Lo monte y, en su investigación "Desarrollo Científico en Costa Rica", originado en los estudios publicados por autores de instituciones costarricenses incluidos en el Siente Citación Índex (Instituye foro Científica Información, Philadelphia, E.U.A.), durante $1980-1998$. El 39,2\% de las publicaciones son del área biomética, $62,3 \%$ son estudiantes colaboradores con instituciones extranjeras, $10 \%$ (189 referentes bibliográficas), pertenecen a la CCSS. Ninguno de los 126 estudios clínicos consignados en los archivos de la Unidad de Investigación y Bioética de la CCSS, están incluidos en este $10 \%$ (25).

\section{DISCUSIÓN}

El estudio y la enseñanza formal de la bioética no es todavía una realidad en las universidades de nuestro país.

Es un deber ineludible que no se debe posponer más, el incorporar el estudio de la bioética en los currículos de los estudiantes de las ciencias médicas y sociales de nuestras universidades públicas y privadas.

En necesario desarrollar en los alumnos y profesionales que van a decidir sobre el bienestar de un paciente y van a integrar comités de bioética, la independencia de criterio que les permita darse cuenta de que no se puede ser juez y parte. Los miembros vinculados de cualquier forma con un 
protocolo de investigación clínica, deben ser excluidos desde el momento que se presenta un protocolo, hasta la publicación de los resultados.

La elección de los miembros de un comité de bioética debe considerar, no sólo experiencia, desempeño profesional, formación bioética, sino también independencia de parentesco, de afiliación política, vínculo comercial o de otra naturaleza, que produzca conflicto de intereses en perjuicio de los seres humanos enfermos.

Es preocupante que, tanto en los predios universitarios, como en los asistentes de la CCSS y el Ministerio de Salud, no se haya creado conciencia sobre la importancia de la bioética y la necesidad de estudiarla y observarla. El deterioro institucional y moral que prevalece en el país, es incompatible con la ciencia al servicio de la sociedad. El sector salud se está saturando de profesionales cuyo móvil principal pareciera ser el lucro y el comercio de la salud y de la ciencia, más que el bienestar social.

Creemos que la bioética no debe estar bajo control político, ni bajo el control de empresas privadas multinacionales fabricantes de medicamentos. La tentación de suprimir todo intento de crear un pensamiento moral beneficioso para los costarricenses es demasiado grande. Si las organizaciones responsables de la salud no ponen límites y se enfrentan a ciertos poderosos grupos de presión económica, no están cumpliendo con su finalidad.

En el umbral del nuevo milenio, el vigoroso desarrollo de la técnica no puede hacernos olvidar que el objetivo de la ciencia es y debe ser el hombre y en consecuencia tiene que ser guiado por la ética. De lo contrario, estaríamos ante un progreso sin orientación y sin norte. 
El desarrollo de la bioética en Costa Rica sólo será útil si se mantiene al margen de intereses comerciales, encubiertos, si se tiene libertad para propugnar nuevas opciones morales, aunque conlleven a la pérdida de dividendos económicos y políticos, para empresas y personas. 


\section{DOCUMENTOS CONSULTADOS}

1. Asociación Médica Mundial. Declaration of Helsinki: recommendations guiding physicians in biomedicals research involving human sujects, 1964.

2. Asociación Médica Mundial. Draft Code of Ethis on Human Experimentation. Br. Med. J. 2 (27 de octubre): 1.119, 1962.

3. Caja Costarricense de Seguro Social, Documentos del Taller de Bioética, Investigación y Desarrollo. Cendeiss, San José, Costa Rica, 1996.

4. CCSS. Informe Auditoría AHC-125-R-2000. Actas Junta Directiva, Sesión 7456, 3 julio 2000, San José, Costa Rica.

5. Códigos Internacionales de Etica. Bol. of Sanit. Panam. 108 (5. 6). 1990.

6. Consejo de Europa. Convenio para la protección de los Derechos Humanos y la dignidad del ser humano con respecto a las aplicaciones de la biología y la medicina. Oviedo, Asturias (España). 1997.

7. Consejo de Organizaciones Internacionales de las Ciencias Médicas (CIOMS), OMS. Pautas Éticas Internacionales para la Investigación y Experimentación Biomédica en Seres Humanos. Ginebra, 1993.

8. Consejo de Organizaciones Internacionales de las Ciencias Médicas (CIOMS), OMS. Pautas Intemacionales para la Evaluación Ética de los Estudios Epistemológicos, Ginebra, 1991.

9. Esquivel, J.M. La bioética y la Declaración Universal de los Derechos Humanos. EDNASSSW. 2000, San José, Costa Rica.

10. Esquivel, J.M. Archivos Unidad de Investigación y Bioética, CCSS, San José, Costa Rica, 1999.

11. Esquivel, J.M. Fundamentos de Bioética, Cendeiss. CCSS, San José, Costa Rica. 1998. 
12. Esquivel, J.M. Normas Institucionales para la Investigación y Experimentación en seres humanos. Dirección Ejecutiva. Cendeiss, CCSS, San José, Costa Rica. 1996.

13. Esquivel, J.M. Programa de Bioética, Dirección Ejecutiva Cendeiss, CCSS, San José, Costa Rica. 1996.

14. Ethics Working Party, European Forum of Good Clinical Practice (EFGCP). Directrices y recomendaciones para las comisiones éticas europeas, 1995. Cuadernos de Bioética (28) 4, 1996.

15. Frenk, J. et al. Un modelo conceptual para la investigación en Salud Pública. Bol. of Sanit. Panam. 101 (5), 1986.

16. Junta Directiva, CCSS. Reglamento para la investigación en los servicios asistentes de la Caja Costarricense de Seguro Social. Acuerdo sesión 7211, 24 marzo 1998. Costa Rica.

17. Kottow, M. Introducción a la Bioética. Editorial Universitaria. Universidad de Chile, OPS, 1995.

18. Laporte, J.R. Principios Básicos de Investigación Clínica. Universidad Autónoma de Barcelona. Edición Ergon S.A., Madrid, 1993.

19. Potter, V.R. Bioethics: bridge to the future. Englewood Clifts, Prentice Hall, 1971.

20. Ramírez, R.; Alfaro M. Ética, Ciencia y Tecnología. 4 Edición, Cartago, Editorial Tecnológico de Costa Rica, 1999.

21. Real Decreto 561-1993, Requisitos para la realización de ensayos clínicos con medicamentos. Ministerio de Sanidad y Consumo. España. Abril, 1993.

22. Rodríguez, M. La tutela constitucional del derecho a la vida y los actos de experimentación sobre seres humanos. Tesis de grado. Universidad de Costa Rica, 1996.

23. Teel, K. The physician's dilema; a doctor's view: what the law should be. Baylor Law Rev. 
24. U.S.A. Government, Code of Federal Regulation. Food and drug. 300-499, Washington, 1995.

25. Sgreccia, Elio. Manual de Bioética. Instituto de Humanismo en Ciencias de la Salud. Universidad Anahuac. Editorial Diana, México, 1996.

26. Lomonte, B. et al. Desarrollo Cientifico en Costa Rica. http:cariari.ucr.ac.cr/blomonte/index.html9/12/1999. 